\title{
Instability in a minimal bimetric gravity model
}

\author{
Frank Koennig ${ }^{1}$, Luca Amendola ${ }^{1}$ \\ ${ }^{1}$ Institut Für Theoretische Physik, Ruprecht-Karls-Universität Heidelberg, Philosophenweg 16, 69120 Heidelberg, Germany
}

\begin{abstract}
We discuss in detail a particularly simple example of a bimetric massive gravity model which seems to offer an alternative to the standard cosmological model at background level. For small redshifts, its equation of state is $w(z) \approx-1.22_{-0.02}^{+0.02}-0.64_{-0.04}^{+0.05} z /(1+z)$. Just like $\Lambda$ CDM, it depends on a single parameter, has an analytical background expansion law and fits the expansion cosmological data well. However, confirming previous results, we find that the model is unstable at early times at small scales and speculate over possible ways to cure the instability. In the regime in which the model is stable, we find that it fits the linear perturbation observations well and has a growth index of approximately $\gamma=0.47$.
\end{abstract}

\section{INTRODUCTION}

The history of massive gravity dates back to 1939, when the linear model of Fierz and Pauli was published (see e.g. Refs. [1] and 2] for a review). Massive gravity requires the introduction of a second tensor field in addition to the metric (or some form of nonlocality in the action; see Ref. [3]). The interaction of the two tensor fields creates a mixture of massless and massive gravitons that apparently avoids the appearance of ghosts [4 7.

In the model introduced in Refs. [8, 9], the second tensor field becomes dynamical, just like the standard metric, although only the latter is coupled to matter (for a generalization, see Ref. [10]). This approach, denoted bimetric gravity, keeps the theory ghosts free and has the advantage of allowing cosmologically viable solutions. The cosmology of bimetric gravity has been studied in several papers, e.g. in Refs. [11 17.

In this paper we select among the class of bimetric models a particularly simple case, which we dub the minimal bimetric model (MBM). Just like $\Lambda \mathrm{CDM}$, this model depends on a single parameter and has an analytical background behavior that is at all times distinguishable from $\Lambda \mathrm{CDM}$. In a previous paper we have shown that the MBM is the only one-parameter version of bimetric gravity (beside the trivial case in which only a cosmological constant is left) that is cosmologically well behaved at the background level and fits the supernovae Hubble diagram well [18] (see also Refs. [11, 12]).

Unfortunately, considering the full set of equations beyond the quasistatic limit, we find that the model is unstable at large wave numbers $k$ in the past and up to a redshift of order unity. This instability has been discussed previously by other authors for bimetric models in general [13, 19] and, if taken at face value, would rule out the model. Nevertheless, we believe it is worth analytically identifying the epoch in which the instability takes place and discussing possible ways to overcome it. This could help to find other cases, within the class of bimetric models, that do not suffer from the same problem.

In the regime in which the model is stable we derive its scalar cosmological perturbation equations in the subhorizon limit and integrate them numerically. We then compare the results with a recent compilation of growth data [20. We find that the MBM fits both supernovae and growth rate data, while remaining well distinguishable from $\Lambda \mathrm{CDM}$. If a variant of the model is found that cures the instability in the past, the model could be an interesting competitor to $\Lambda \mathrm{CDM}$.

\section{BACKGROUND EQUATIONS}

We start with the action of the form [8]

$$
\begin{aligned}
S & =-\frac{M_{g}^{2}}{2} \int d^{4} x \sqrt{-g} R(g)-\frac{M_{f}^{2}}{2} \int d^{4} x \sqrt{-f} R(f) \\
& +m^{2} M_{g}^{2} \int d^{4} x \sqrt{-g} \sum_{n=0}^{4} \beta_{n} e_{n}(X)+\int d^{4} x \sqrt{-g} L_{m}
\end{aligned}
$$

where $X_{\gamma}^{\alpha} \equiv \sqrt{g^{\alpha \beta} f_{\beta \gamma}}, e_{n}$ are elementary symmetric polynomials, $\beta_{n}$ are arbitrary constants and $L_{m}=L_{m}(g, \psi)$ is a matter Lagrangian. Here $g_{\mu \nu}$ is the standard metric coupled to matter fields in the $L_{m}$ Lagrangian, while $f_{\mu \nu}$ is an additional dynamical tensor field. In the following we express masses in units of the Planck mass $M_{g}$ and the mass parameter $m^{2}$ will be absorbed into the parameters $\beta_{n}$. Varying the action with respect to $g_{\mu \nu}$, one obtains the 
following equations of motion:

$$
G_{\mu \nu}+\frac{1}{2} \sum_{n=0}^{3}(-1)^{n} \beta_{n}\left[g_{\mu \lambda} Y_{(n) \nu}^{\lambda}(X)+g_{\nu \lambda} Y_{(n) \mu}^{\lambda}(X)\right]=T_{\mu \nu}
$$

where $G_{\mu \nu}$ is Einstein's tensor, and the expressions $Y_{(n) \nu}^{\lambda}(X)$ are defined as

$$
\begin{aligned}
Y_{(0)} & =I \\
Y_{(1)} & =X-I[X] \\
Y_{(2)} & =X^{2}-X[X]+\frac{1}{2} I\left([X]^{2}-\left[X^{2}\right]\right), \\
Y_{(3)} & \left.=X^{3}-X^{2}[X]+\frac{1}{2} X\left([X]^{2}-\left[X^{2}\right]\right)\right) \\
& -\frac{1}{6} I\left([X]^{3}-3[X]\left[X^{2}\right]+2\left[X^{3}\right]\right),
\end{aligned}
$$

where $I$ is the identity matrix and $[\ldots]$ is the trace operator. Varying the action with respect to $f_{\mu \nu}$ we get

$$
\bar{G}_{\mu \nu}+\sum_{n=0}^{3} \frac{(-1)^{n} \beta_{4-n}}{2 M_{f}^{2}}\left[f_{\mu \lambda} Y_{(n) \nu}^{\lambda}\left(X^{-1}\right)+f_{\nu \lambda} Y_{(n) \mu}^{\lambda}\left(X^{-1}\right)\right]=0
$$

where the overbar indicates $f_{\mu \nu}$ curvatures. Notice that $\beta_{0}$ acts as a pure cosmological constant. Finally, the rescaling $f \rightarrow M_{f}^{-2} f, \beta_{n} \rightarrow M_{f}^{n} \beta_{n}$ allows us to assume $M_{f}=1$ in the following (see Ref. [17]).

We assume now a cosmological spatially flat Friedmann-Robertson-Walker (FRW) metric,

$$
d s^{2}=a^{2}(t)\left(-d t^{2}+d x_{i} d x^{i}\right)
$$

where $t$ represents the conformal time and a dot will represent the derivative with respect to it. The second metric is chosen also in a FRW form

$$
d s_{f}^{2}=-\left[\dot{b}(t)^{2} / \mathcal{H}^{2}(t)\right] d t^{2}+b(t)^{2} d x_{i} d x^{i}
$$

where $\mathcal{H} \equiv \dot{a} / a$ is the conformal Hubble function. This form of the metric $f_{\mu \nu}$ ensures that the equations satisfy the Bianchi identities (see e.g. Ref. [9]).

Defining $r=b / a$, the background equations can be conveniently written as a first-order system for $r, \mathcal{H}$, using $N=\log a$ as the time variable and denoting $d / d N$ with a prime [18] (see also [12]):

$$
\begin{aligned}
2 E^{\prime} E+E^{2} & =a^{2}\left(B_{0}+B_{2} r^{\prime}\right), \\
r^{\prime} & =\frac{3 r B_{1} \Omega_{m}}{\beta_{1}-3 \beta_{3} r^{2}-2 \beta_{4} r^{3}+3 B_{2} r^{2}},
\end{aligned}
$$

where $\Omega_{m}=1-\frac{B_{0}}{B_{1}} r, E \equiv \mathcal{H} / H_{0}$ and the couplings $\beta_{i}$ are measured in units of $H_{0}^{2}$ and finally

$$
\begin{aligned}
& B_{0}=\beta_{0}+3 \beta_{1} r+3 \beta_{2} r^{2}+\beta_{3} r^{3} \\
& B_{1}=\beta_{1}+3 \beta_{2} r+3 \beta_{3} r^{2}+\beta_{4} r^{3} \\
& B_{2}=\beta_{1}+2 \beta_{2} r+\beta_{3} r^{2} .
\end{aligned}
$$

\section{MINIMAL BIMETRIC MODEL}

In Ref. [18 we identified the conditions for standard cosmological viability, i.e. for a matter epoch followed by a stable acceleration, without bounces or singularities beside the big bang. We found that among the models with a single nonvanishing parameter only two cases give a viable cosmology, namely, the cases with only $\beta_{0}$ or only $\beta_{1}$. The former one is indeed the $\Lambda \mathrm{CDM}$ model, while the $\beta_{1}$ case is what we call the minimal bimetric model. One has then for the MBM

$$
r^{\prime}=\frac{3 r\left(1-3 r^{2}\right)}{1+3 r^{2}}
$$


independent of $\beta_{1}$. This equation has two branches for $r>0$, but only the one that starts at $r=0$ and ends at $r=1 / \sqrt{3}$ is cosmologically viable. In terms of the scale factor, this solution reads [18, 21]

$$
r(a)=\frac{1}{6} a^{-3}\left(-A \pm \sqrt{12 a^{6}+A^{2}}\right)
$$

where $A=-\beta_{1}+3 / \beta_{1}$. These equations imply a remarkably simple and testable relation between the equation of state $w$ and $\Omega_{m}$ valid at all times during matter domination:

$$
w=\frac{2}{\Omega_{m}-2},
$$

where the density parameter is given by

$$
\Omega_{m}=1-3 r(a)^{2}
$$

Since the Friedmann equation of the second metric provides $r_{0}=\beta_{1} / 3$, the present value of the matter density parameter is therefore simply related to single parameter value of the model. Together with Eq. (17) this shows that all viable parameter values for $\beta_{1}$ lead to a phantom equation of state at present time. Another useful relation for the MBM that we will use below is $\mathcal{H}^{2}=\beta_{1} a^{2} / 3 r$.

In Ref. [18] we found that the MBM fits the supernovae data well if $\beta_{1}=1.38 \pm 0.03$, corresponding to $\Omega_{m 0}=$ $1-\beta_{0}^{2} / 3=0.37 \pm 0.02$. The equation of state turns out to be approximated at small redshifts by $w(z) \approx-1.22_{-0.02}^{+0.02}-$ $0.64_{-0.04}^{+0.05} z /(1+z)$. However this parametrization is not adequate at $z \geq 0.5$ and the analytic expressions 16 - 18 should be employed instead.

\section{PERTURBATION EQUATIONS}

We now find the perturbation equations for the MBM. For the perturbed part of the metrics we adopt the gauge defined in Fourier space as

$$
\begin{aligned}
& d s_{f}^{2}=2 F b^{2}\left[-\frac{\dot{b}(t)^{2} \Psi_{f}}{b(t)^{2} \mathcal{H}^{2}(t)} d t^{2}+\left(\Phi_{f} \delta_{i j}+k_{i} k_{j} E_{f}\right) d x^{i} d x^{j}\right] \\
& d s^{2}=2 F a^{2}\left[-\Psi d t^{2}+\left(\Phi \delta_{i j}+k_{i} k_{j} E\right) d x^{i} d x^{j}\right]
\end{aligned}
$$

where $F=e^{i \mathbf{k} \cdot \mathbf{r}}$. After a transformation to the gauge-invariant variables [13]

$$
\begin{aligned}
\tilde{\Phi} & =\Phi-\mathcal{H}^{2} E^{\prime} \\
\tilde{\Psi} & =\Psi-\left(\mathcal{H}^{2}+\mathcal{H} \mathcal{H}^{\prime}\right) E^{\prime}-\mathcal{H}^{2} E^{\prime \prime} \\
\tilde{\Phi}_{f} & =\Phi_{f}-\frac{r \mathcal{H}^{2} E_{f}^{\prime}}{\left(r^{\prime}+r\right)}, \\
\tilde{\Psi}_{f} & =\Psi_{f}-\frac{\mathcal{H} r^{2}\left(\mathcal{H} E_{f}^{\prime}\right)^{\prime}}{\left(r^{\prime}+r\right)^{2}}-\frac{\mathcal{H}^{2} E_{f}^{\prime} r\left(r^{2}+2 r^{\prime 2}+2 r r^{\prime}-r r^{\prime \prime}\right)}{\left(r^{\prime}+r\right)^{3}}
\end{aligned}
$$

we obtain from the Einstein equations a set of perturbation equations in $\Xi=\left\{\tilde{\Phi}, \tilde{\Psi}_{f} \tilde{\Phi}_{f}, \tilde{\Psi}_{f}, E, \Delta E \equiv E-E E_{f}\right\}$,

$$
\begin{aligned}
& {[00] \Phi\left(1+\frac{2 k^{2}}{3 a^{2} r \beta_{1}}\right)-\Phi_{f}+\frac{a^{2}\left(1-3 r^{2}\right) \beta_{1}}{-4 r+6 r^{3}} E^{\prime}+\frac{\mathcal{H}^{2}\left(1+3 r^{2}\right)}{-4+6 r^{2}} \Delta E^{\prime}+\frac{1}{3} k^{2} \Delta E} \\
& -\frac{a\left(-1+3 r^{2}\right) \sqrt{\beta_{1}}}{\sqrt{3} k^{2} r^{5 / 2}} \theta-\frac{\delta \rho}{3 B_{2} r}=0, \\
& {[0 i] \Phi^{\prime}-\Psi+\frac{a^{2} \rho}{2 \mathcal{H} k^{2}} \theta+\left(\mathcal{H}^{2}-\mathcal{H} \mathcal{H}^{\prime}\right) E^{\prime}=0,} \\
& {[i j] \Phi+\Psi+a^{2} r \beta_{1} \Delta E=0} \\
& {[i c]-\left(2+\frac{2 k^{2}}{3 a^{2} r \beta_{1}}\right) \Phi+2 \Phi_{f}-\Psi\left(1+\frac{2 k^{2}}{3 a^{2} r \beta_{1}}\right)+\frac{6\left(2-3 r^{2}\right)}{3+9 r^{2}} \Psi_{f}+\frac{\mathcal{H}^{3} r\left(3+9 r^{2}\right)\left(\mathcal{H}-\mathcal{H}^{\prime}\right)}{a^{2}\left(2-3 r^{2}\right) \beta_{1}} E^{\prime \prime}+\frac{3 a^{2}\left(2+9 r^{2}\right)\left(1-3 r^{2}\right)^{2} \beta_{1}}{4 r\left(2-3 r^{2}\right)^{2}\left(1+3 r^{2}\right)} E^{\prime}} \\
& -\frac{2 k^{2}}{3} \Delta E+\frac{a^{2}\left(1+3 r^{2}\right) \beta_{1}}{6 r\left(2-3 r^{2}\right)} \Delta E^{\prime \prime}+\frac{a^{2}\left(22-9 r^{2}\left(-19+42 r^{2}+15 r^{4}\right)\right) \beta_{1}}{12 r\left(4-27 r^{4}+27 r^{6}\right)} \Delta E^{\prime}=0,
\end{aligned}
$$




$$
\begin{aligned}
& {[00] \Phi+\left(-1-\frac{2 k^{2} r}{3 a^{2} \beta_{1}}\right) \Phi_{f}+\frac{k^{2}}{3} \Delta E+\frac{a^{2}\left(-1+3 r^{2}\right) \beta_{1}}{4 r-6 r^{3}} E^{\prime}-\frac{a^{2}\left(1+3 r^{2}\right) \beta_{1}}{6 r\left(2-3 r^{2}\right)} \Delta E^{\prime}=0,} \\
& {[0 i] \Phi_{f}^{\prime}+\frac{\left(-4+6 r^{2}\right)}{1+3 r^{2}} \Psi_{f}+\frac{3 a^{2}\left(-1+3 r^{2}\right) \beta_{1}}{4 r\left(-2+3 r^{2}\right)} E^{\prime}+\frac{3 a^{2}\left(1-3 r^{2}\right) \beta_{1}}{4 r\left(-2+3 r^{2}\right)} \Delta E^{\prime}=0,} \\
& {[i j] \Psi_{f}+\Phi_{f}+\frac{a^{2}\left(1+3 r^{2}\right) \beta_{1}}{-4 r+6 r^{3}} \Delta E=0,} \\
& {[i i] \Phi+\left(-1+\frac{2 k^{2} r\left(-2+3 r^{2}\right)}{3 a^{2}\left(1+3 r^{2}\right) \beta_{1}}\right) \Phi_{f}+\frac{\Psi}{2}+\left(1-\frac{3}{1+3 r^{2}}+\frac{2 k^{2} r\left(-2+3 r^{2}\right)}{3 a^{2}\left(1+3 r^{2}\right) \beta_{1}}\right) \Psi_{f}+\frac{a^{2}\left(1-3 r^{2}\right) \beta_{1}}{4 r\left(-2+3 r^{2}\right)} E^{\prime \prime}+\frac{a^{2}\left(1+3 r^{2}\right) \beta_{1}}{12 r\left(-2+3 r^{2}\right)} \Delta E^{\prime \prime}} \\
& \quad+\frac{1}{3} k^{2} \Delta E+\frac{a^{2}\left(-22+9 r^{2}\left(-19+42 r^{2}+15 r^{4}\right)\right) \beta_{1}}{24 r\left(4-27 r^{4}+27 r^{6}\right)} \Delta E^{\prime}-\frac{3\left(2+9 r^{2}\right)\left(a-3 a r^{2}\right)^{2} \beta_{1}}{8\left(2-3 r^{2}\right)^{2}\left(r+3 r^{3}\right)} E^{\prime}=0,
\end{aligned}
$$

and from the conservation of matter we get two more equations for the matter density contrast $\delta$ and the velocity divergence $\theta$,

$$
\begin{aligned}
& \delta^{\prime}+\theta \mathcal{H}^{-1}+3 \Phi^{\prime}-3 \mathcal{H}^{2} E^{\prime \prime}-6 \mathcal{H} \mathcal{H}^{\prime} E^{\prime}+k^{2} E^{\prime}=0, \\
& \theta^{\prime}+\theta+k^{2} E^{\prime} \mathcal{H}^{\prime}-k^{2} \Psi \mathcal{H}^{-1}+k^{2} \mathcal{H}\left(E^{\prime \prime}+E^{\prime}\right)=0 .
\end{aligned}
$$

\section{INSTABILITY}

Recently some authors [13, 19] found an instability at small scales in massive bimetric theories. Here we revisit this issue in the MBM. Starting from the set of general perturbation equations (24)-(31), one can replace all $\Psi_{f}, \Phi_{f}, \Delta E$ and their derivatives by using $g_{00}, g_{i i}$ and $g_{i j}$. This also shows that eqs $g_{i j}$ and $f_{i j}$ are linearly dependent. Then we can replace $\delta$ and $\theta$ with the help of $g_{0 i}$ and $f_{00}$. Finally, one can find a linear combination of $f_{0 i}$, and $g_{i i}$ which allows one to express $E^{\prime}$ as a function of $\Psi, \Phi$ and their derivatives. In this way, we can express our original ten equations to just two second-order equations for $X \equiv\{\Psi, \Phi\}$ which can be written as $(i, j=1,2)$

$$
X_{i}^{\prime \prime}+M_{i j} X_{j}^{\prime}+N_{i j} X_{j}=0
$$

where $M_{i j}$ and $N_{i j}$ are two matrices that depend only on $k, \beta_{1}$ and $r$. For the explicit expressions of their elements see Appendix A. The eigenfrequencies of this equation can be found by substituting $X=X_{0} e^{i \omega N}$, assuming that the dependence of $\omega$ on time is negligibly small. In the limit of large $k$ we find

$$
\omega_{\mp}= \pm \frac{k}{\mathcal{H}} \frac{\sqrt{-1+12 r^{2}+9 r^{4}}}{1+3 r^{2}}
$$

(here $k$ is in the same units as $\mathcal{H}$ ) plus two other solutions, one of which is zero while the is independent of $k$ and therefore subdominant. One can then see that real solutions (needed to obtain an oscillating, rather than a growing, solution for $X$ ) are found only for $r>0.28$, which occurs for $N \approx-0.4$, i.e. $z \approx 0.5$. This is exactly the same instant at which $r^{\prime \prime}$ crosses zero. At any epoch before this, the perturbation equations are unstable for large $k$, i.e. they grow as $a^{\omega_{+}}$. Notice that $\omega_{\mp}$ are independent of $\beta_{1}$; this means that the instability remains even in the limit of zero mass, which is similar to the van Dam-Veltman-Zakharov discontinuity [22, 23]. Similar to that case, one might speculate that when nonlinear order effects start being important they might cure the instability. Notice also that the large- $k$ limit we have taken is valid only for $k / \mathcal{H} \gg 1$, i.e. for $r>r_{H}$, where $r_{H}(k)$ is the solution of the equation $a(r)^{2}=3 r k^{2} / \beta_{1}$ and $a(r)$ is obtained by inverting Eq. 16.

This explosively large growth is in obvious contrast with what we know about the growth of linear perturbations in our Universe, for instance, with the smoothness of the microwave cosmic background and the linearity of present fluctuations on scales larger than a few megaparsecs. However, one might imagine that by adjusting for instance the initial conditions or by playing with other assumptions, the model could be saved. Therefore, in order to quantify the real impact of the instability, we estimate a directly observable quantity that is independent of initial conditions: the growth rate of the linear perturbations as measured with redshift distortions. Since all the perturbation variables can be written as a linear combination of $\Phi$ and $\Psi$, their dominant behavior will have the same growth $\sim e^{i \omega_{+} N}$. This means that during the instability epoch the matter density contrast grows as $\delta \sim a^{\omega}$ where $\omega=\left|\omega_{+}\right|$. This allows us to estimate the growth rate $f \equiv d \log \delta / d N$ and to obtain the observable combination $f(z) \sigma_{8}(z)=\sigma_{8} f \delta / \delta_{0}$ as

$$
f(z) \sigma_{8}(z)=A a^{\omega}\left(\omega+N \omega^{\prime}\right)
$$


where $A$ is a normalization constant. The combination $f \sigma_{8}(z)$ has been estimated through redshift distortions at various redshifts up to unity (see for instance Ref. [20]), and it has been found to be practically constant in the range from $z=0.8$ to $z=0.3$ for scales around $k=0.1 \mathrm{~h} / \mathrm{Mpc}$, corresponding to $k / H_{0} \approx 50$. In stark contrast, using the expression (36), we estimate an extremely fast growth during the instability epoch; for instance, between $z=0.8$ and $z=0.6$ the growth of $f \sigma_{8}(z)$ is found to be around 180,000 times.

Adding the cosmological constant $\beta_{0}$, one obtains

$$
\omega_{\mp}= \pm \frac{k}{\mathcal{H}} \frac{\sqrt{-1+2\left(\beta_{0} / \beta_{1}\right) r+12 r^{2}+9 r^{4}}}{1+3 r^{2}} .
$$

In this case the instability region occurs for any $r<\beta_{1} / 2 \beta_{0}$; if $\beta_{1} / \beta_{0} \ll 1$ this unstable epoch can be pushed arbitrarily back into the past but then the model would effectively behave like $\Lambda$ CDM.

It is possible that a different choice of parameters $\beta_{i}$ leads to an evolution which is free from instabilities, or a value of $r_{H}(k)$ such that (at least for the scales that are today in the linear regime) the subhorizon evolution occurs during the stable phase. Finally, one could also assume that $\beta_{1}$ is actually a time-dependent variable (e.g., it could be a function of a scalar field, $\left.\beta_{1}(\phi)\right)$, so that its value is very small in the past - therefore recovering a standard evolution - and comparable to $H_{0}$ near the present epoch.

\section{QUASISTATIC LIMIT}

Taken at face value, the instability rules out the MBM, unless nonlinear effects are able to rescue it. However, we think it is still worthwhile to consider some of its cosmological effects for two reasons. First, one of the mentioned mechanisms or some variants thereof might be able to cure the past instability while leaving unaltered the recent epoch. Second, the methods we investigate below can be applied to other choices of parameters in the bimetric class that allow for a stable evolution.

In the regime in which the model is stable, i.e. for $z \leq 0.5$, one can simplify the perturbation equations by taking the quasistatic limit. In this regime and at subhorizon scales, i.e. $k / \mathcal{H} \gg 1$, we can in fact assume that $\Xi_{i}(k / \mathcal{H})^{2}$ is much larger than $\Xi_{i}$ and its derivatives $\Xi_{i}^{\prime}, \Xi_{i}^{\prime \prime}$ for any $\Xi_{i}=\left\{\Phi, \Psi, \Phi_{f}, \Psi_{f}, \Delta E, E\right\}$ and also $\delta(k / \mathcal{H})^{2}, \delta^{\prime}(k / \mathcal{H})^{2} \gg \theta / \mathcal{H}$; then the set of differential equations becomes algebraic (except for the matter conservation equations) and we obtain the Poisson-like relations

$$
\begin{gathered}
\Psi=-\frac{\mathcal{H}^{2} \Omega_{m} \delta\left(2 k^{2} r^{3}\left(11+6 r^{2}\right)+3 \beta_{1} a^{2}\left(1+7 r^{2}-6 r^{4}\right)\right)}{2 k^{2}\left(\beta_{1} a^{2}\left(1+r^{2}\right)^{2}\left(1+3 r^{2}\right)+k^{2} r^{3}\left(7+3 r^{2}\right)\right)}, \\
\Phi=\frac{\mathcal{H}^{2} \Omega_{m} \delta\left(2 k^{2} r^{3}\left(10+3 r^{2}\right)+3 \beta_{1} a^{2}\left(1+4 r^{2}+3 r^{4}\right)\right)}{2 k^{2}\left(\beta_{1} a^{2}\left(1+r^{2}\right)^{2}\left(1+3 r^{2}\right)+k^{2} r^{3}\left(7+3 r^{2}\right)\right)}, \\
\Psi_{f}=-\frac{\mathcal{H}^{2} \Omega_{m} \delta\left(3 r^{2}+1\right)\left(3 \beta_{1} a^{2}\left(6 r^{4}-7 r^{2}-1\right)+2 k^{2} r\left(6 r^{2}-1\right)\right)}{4 k^{2}\left(3 r^{2}-2\right)\left(\beta_{1} a^{2}\left(r^{2}+1\right)^{2}\left(3 r^{2}+1\right)+k^{2}\left(3 r^{2}+7\right) r^{3}\right)}, \\
\Phi_{f}=\frac{\mathcal{H}^{2} \Omega_{m} \delta\left(3 r^{2}+1\right)\left(3 \beta_{1} a^{2}\left(r^{2}+1\right)+k^{2} r\right)}{2 k^{2}\left(\beta_{1} a^{2}\left(r^{2}+1\right)^{2}\left(3 r^{2}+1\right)+k^{2}\left(3 r^{2}+7\right) r^{3}\right)}, \\
\Delta E=\frac{\mathcal{H}^{2} \Omega_{m} \delta r\left(9 \beta_{1} a^{2}\left(1-3 r^{2}\right)+2 k^{2} r\left(3 r^{2}+1\right)\right)}{2 a^{2} \beta_{1} k^{2}\left(\beta_{1} a^{2}\left(r^{2}+1\right)^{2}\left(3 r^{2}+1\right)+k^{2}\left(3 r^{2}+7\right) r^{3}\right)},
\end{gathered}
$$

which reduce to the standard ones during the matter epoch, i.e. for $r \rightarrow 0$. In the quasistatic limit the set of equations does not contain the $(0, \mathrm{i}) g_{\mu \nu}$ and $(0, \mathrm{i}) f_{\mu \nu}$ equations. Since both equations were used to simplify the remaining ones, we numerically have checked the consistency of the solutions with both $(0, \mathrm{i})$ equations. We then obtain the two modified gravity parameters

$$
\begin{aligned}
\eta & \equiv-\frac{\Phi}{\Psi}=H_{2} \frac{1+H_{4}(k / \mathcal{H})^{2}}{1+H_{3}(k / \mathcal{H})^{2}} \\
Y & \equiv-\frac{2 k^{2} \Psi}{3 \mathcal{H}^{2} \Omega_{m} \delta_{m}}=H_{1} \frac{1+H_{3}(k / \mathcal{H})^{2}}{1+H_{5}(k / \mathcal{H})^{2}}
\end{aligned}
$$


where

$$
\begin{aligned}
H_{1} & \equiv \frac{1+7 r^{2}-6 r^{4}}{\left(1+r^{2}\right)^{2}\left(1+3 r^{2}\right)}, \\
H_{2} & \equiv \frac{1+4 r^{2}+3 r^{4}}{1+7 r^{2}-6 r^{4}}, \\
H_{3} & \equiv \frac{2 \mathcal{H}^{2} r^{3}\left(11+6 r^{2}\right)}{3 \beta_{1} a^{2}\left(1+7 r^{2}-6 r^{4}\right)}, \\
H_{4} & \equiv \frac{2 \mathcal{H}^{2} r^{3}\left(10+3 r^{2}\right)}{3 \beta_{1} a^{2}\left(1+4 r^{2}+3 r^{4}\right)}, \\
H_{5} & \equiv \frac{\mathcal{H}^{2} r^{3}\left(7+3 r^{2}\right)}{\beta_{1} a^{2}\left(1+r^{2}\right)^{2}\left(1+3 r^{2}\right)} .
\end{aligned}
$$

For $\beta_{1} \rightarrow 0$ the only consistent background solution is $r \rightarrow 0$; in this limit the model reduces to pure CDM and consequently $H_{1,2}=1$ and $H_{3,4,5}=0$. The expressions $(43)$ and (44) have the same structure as the Horndeski Lagrangian [24 26] since both Lagrangians produce second-order equations of motion. The matter evolution equations can now be written as a single equation:

$$
\delta_{m}^{\prime \prime}+\delta_{m}^{\prime}\left(1+\frac{\mathcal{H}^{\prime}}{\mathcal{H}}\right)-\frac{3}{2} Y(k) \Omega_{m} \delta_{m}=0 .
$$

Integrating numerically this equation along the background solution (16), we find that near $k=0.1 h / \mathrm{Mpc}$ and $\beta_{1}=1.39$ we can approximate $f \equiv \delta^{\prime} / \delta \approx \Omega_{m}^{\gamma}$ [27] with $\gamma \approx 0.47$ in the range $z \in(0,5)$ (see Fig. 11. Near $\beta_{1}=1.39$ the dependence on $\beta_{1}$ at $k=0.1 \mathrm{~h} / \mathrm{Mpc}$ can be linearly approximated as $\gamma=0.26+0.15 \beta_{1}$, while the weak dependence on $k$ is approximately

$$
\gamma(k)=0.47+0.001\left(\frac{k}{0.1 h / \mathrm{Mpc}}\right)^{-1 / 2} .
$$

Future experiments, like the Euclid satellite [28], plan to measure $\gamma$ to within 0.02 ; this will amply allow one to distinguish the MBM from $\Lambda \mathrm{CDM}$ and standard quintessence, which predict $\gamma \approx 0.54$.

Let us remark, however, that the growth rate is significantly larger than 1 for redshifts $z \gtrsim 1$ and cannot be well approximated with the standard $\Omega_{m}^{\gamma}$ fit. We find that an additional correction

$$
f \approx \Omega_{m}^{\gamma_{0}}\left(1+\frac{\gamma_{1}}{z+1}\right)
$$

with $\gamma_{0}=0.58$ and $\gamma_{1}=0.07$ is better able to reproduce our numerical result.

The quasistatic limit is an excellent approximation to the full behavior, provided one considers only the stable epoch $z<0.5$, as shown in Fig. 2 .

\section{COMPARISON TO THE GROWTH RATE}

The quasistatic limit can be compared to measurements of $f(z) \sigma_{8}(z)$ where $\sigma_{8}(z)=\sigma_{8} G(z)$, with $G(z)$ being the growth rate normalized to unity today. Most of the present measurements actually extend to redshifts higher than 0.5 , which is outside the stability regime. Nevertheless, as a way to demonstrate the feasibility of constraining this model with growth data, we include these measurements as well. The likelihood is given by

$$
\chi_{f \sigma_{8}}^{2}=\sum_{i j}\left(d_{i}-\sigma_{8} t_{i}\right) C_{i j}^{-1}\left(d_{j}-\sigma_{8} t_{j}\right),
$$

in which $d_{i}$ and $t_{i}$ are vectors containing the measured and theoretically expected data, respectively, and $C_{i j}$ denotes the covariance matrix. Since the current constraints on $\sigma_{8}$ depend on the theory of gravity, for generality we marginalize analytically the likelihood over $\sigma_{8}>0$, obtaining

$$
\chi_{f \sigma_{8}}^{2}=S_{20}-\frac{S_{11}^{2}}{S_{02}}+\log S_{02}-2 \log \left(1+\operatorname{Erf}\left(\frac{S_{11}}{\sqrt{2 S_{02}}}\right)\right),
$$




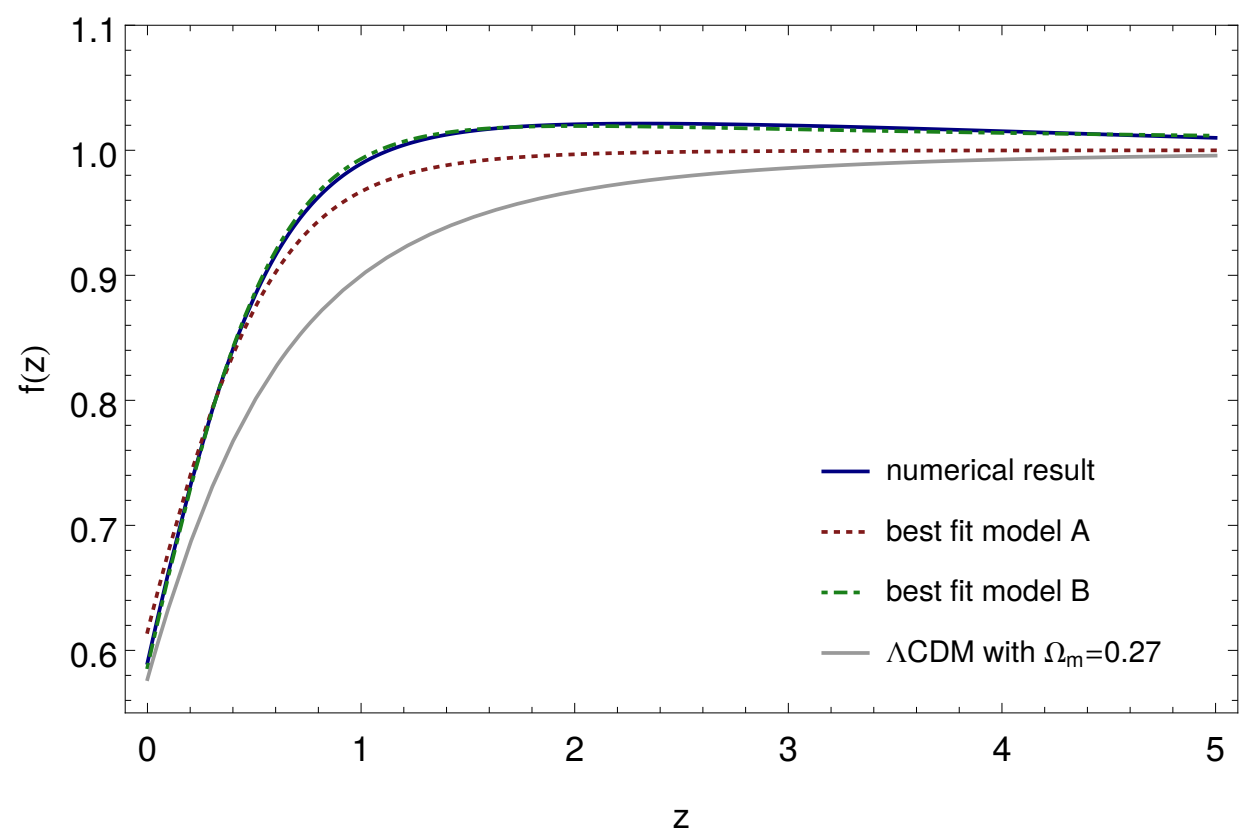

Figure 1: Growth rate $f=\delta^{\prime} / \delta$ in the quasistatic limit for $\beta_{1}=1.39$ and $k=0.1 h /$ Mpc. The numerical result (in blue) is approximated by the fitting model $f=\Omega_{m}^{\gamma}$ (model A, red dotted curve) and $f=\Omega_{m}^{\gamma_{0}}\left(1+\frac{\gamma_{1}}{z+1}\right)$ (model B, green dash-dotted curve). For a comparison we plot the $\Lambda$ CDM result (gray dashed line) while using $\Omega_{m 0}=0.37$ which corresponds to the present matter density in our analyzed MBM.

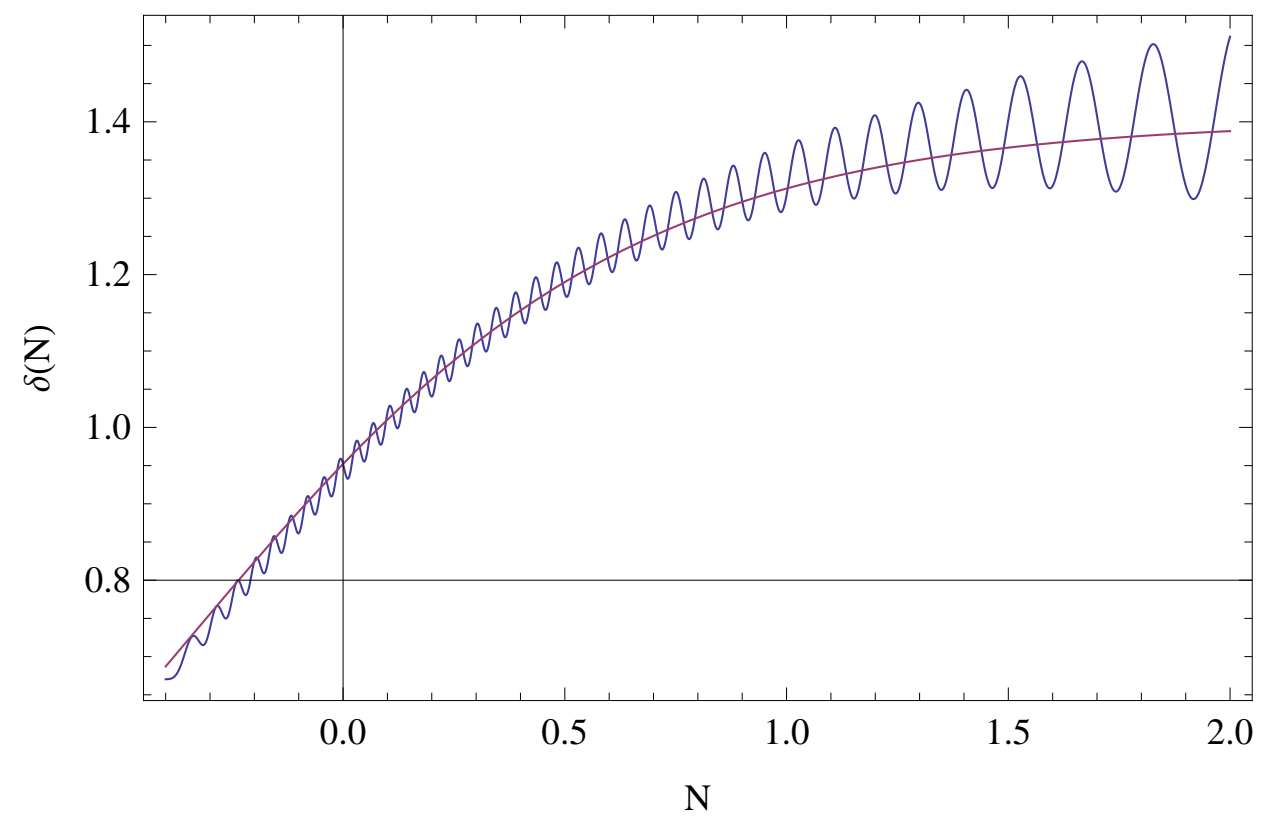

Figure 2: The oscillating blue line represents the numerical integration of the full set of perturbation equations for $k / H_{0}=$ $100, \beta_{1}=1.4$. The red smooth line is the solution of the growth equation 50 in the quasistatic limit for the same parameters.

where

$$
\begin{aligned}
& S_{11}=d_{i} C_{i j}^{-1} t_{j}, \\
& S_{20}=d_{i} C_{i j}^{-1} d_{j}, \\
& S_{02}=t_{i} C_{i j}^{-1} t_{j}
\end{aligned}
$$




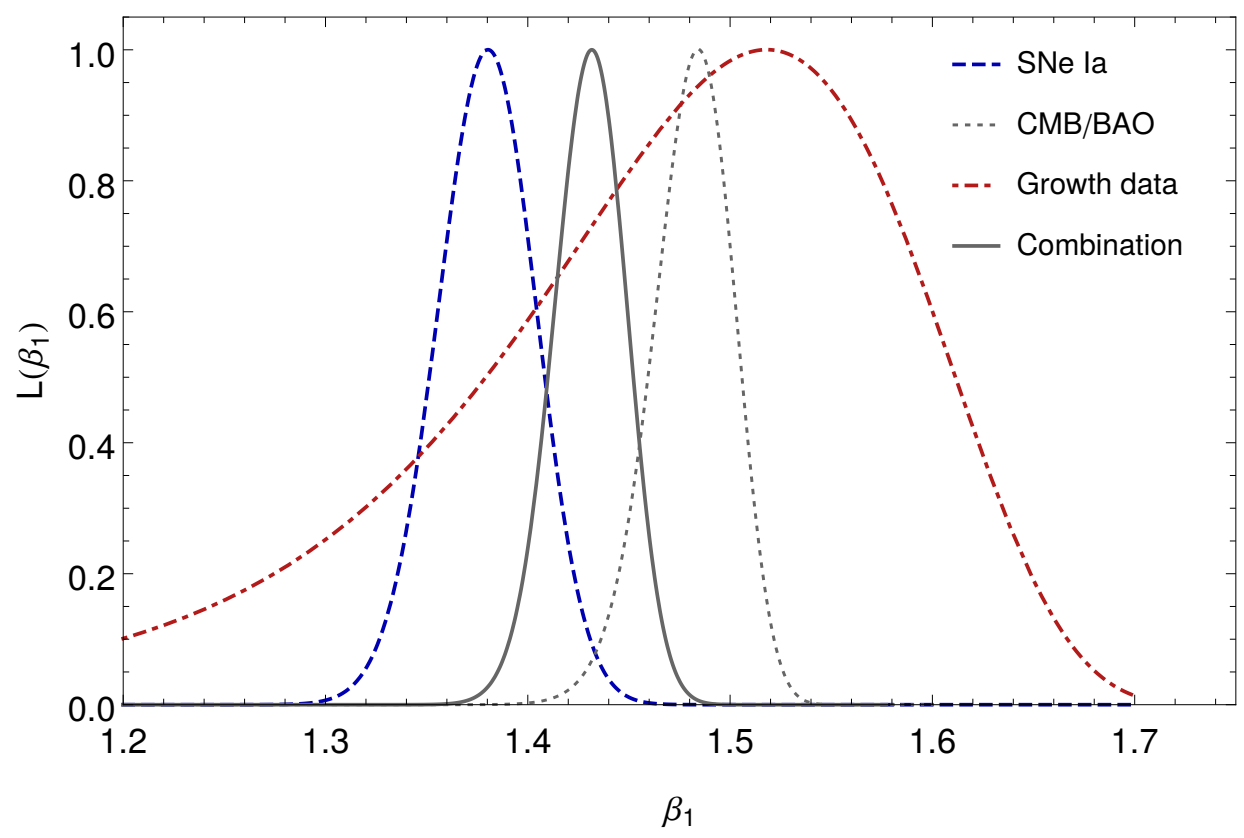

Figure 3: Likelihood for $\beta_{1}$ obtained from observed SNe Ia (blue dashed), measured growth data (red dot-dashed) and the combination of CMB and BAO measurements (dotted gray). The full combined likelihood is indicated by a gray solid line. All likelihoods are rescaled to unity at their maximum. For the most likely values we obtain $\beta_{1}=1.38_{-0.03}^{+0.03}\left(\chi_{\text {min }}^{2}=578.3\right)$ and $\beta_{1}=1.52_{-0.13}^{+0.09}\left(\chi_{\min }^{2}=10.48\right)$ for the comparison with SNe Ia and growth data, respectively. Due to the broad width of the growth likelihood, its combination with the other probes does not sensibly change the results.

(see also e.g. Ref. [29]). Since current data are not binned in $k$ space, we choose an average value $k=0.1 h / \mathrm{Mpc}$ in Eq. (50).

We compute the likelihood from the data set compiled in [20] which contains measured growth histories from the 6dFGS [30, $\mathrm{LRG}_{200}, \mathrm{LRG}_{60}$ 31], BOSS [32, WiggleZ [33] and VIPERS 34] surveys. Our results are shown in Fig. 3. The growth data constraints appear much broader than, but consistent with, the supernova type Ia (SN Ia) data. The combined result from $\mathrm{SNe}$ and growth data is $\beta_{1}=1.39 \pm 0.03$, practically identical to the best fit from SN Ia alone. We also plot in Fig. 3 the likelihood from cosmic microwave background (CMB) and baryon acoustic oscillation (BAO) measurements where we use the results from the first peak angular size WMAP 7.2 data [35] and the SDSS DR7 sample including the LRG and 2dFGRS data set [36]. The combined result from all data, SN + CMB + BAO + growth turns out to be $\beta_{1}=1.43 \pm 0.02$. However, one should keep in mind that the CMB data analysis assumes a pure $\Lambda \mathrm{CDM}$ so it is not obvious that it can be applied here without corrections. Note that including the CMB/BAO data does not change the best-fit parameters for $w(z)$ and $\gamma$ significantly.

Finally, in Fig. 4 we compare the growth history corresponding to the most likely MBM with the measured growth data and the $\Lambda \mathrm{CDM}$ expectation.

\section{CONCLUSIONS}

We have shown that a minimal bimetric model exists which closely reproduces the success and the simplicity of $\Lambda \mathrm{CDM}$ at the background level. We fixed its single parameter, $\beta_{1}$, to percent accuracy by fitting to supernovae and growth data. The MBM has several unique signatures, like the $w-\Omega_{m}$ relation (17), the phantom equation of state, the $k$ dependence of the growth factor (Eq. 51) and the values of $f$ above unity (Eq. 52), all of which will make it easily distinguishable from $\Lambda$ CDM with future experiments.

We have shown however that the model suffers from a perturbation instability at large $k$ at epochs before $z \approx 0.5$, confirming previous results [13, 19] but also identifying the exact epoch of transition. Taken at face value, such an instability seems to rule out this particular form of bimetric model. A possible way to save the model is to assume that when the perturbations become nonlinear the instability becomes under control. This conjecture can be confirmed only by going to higher order in perturbation theory. Of course the instability might also disappear by choosing a different set of parameters. We leave a complete analysis of other models to future work. 


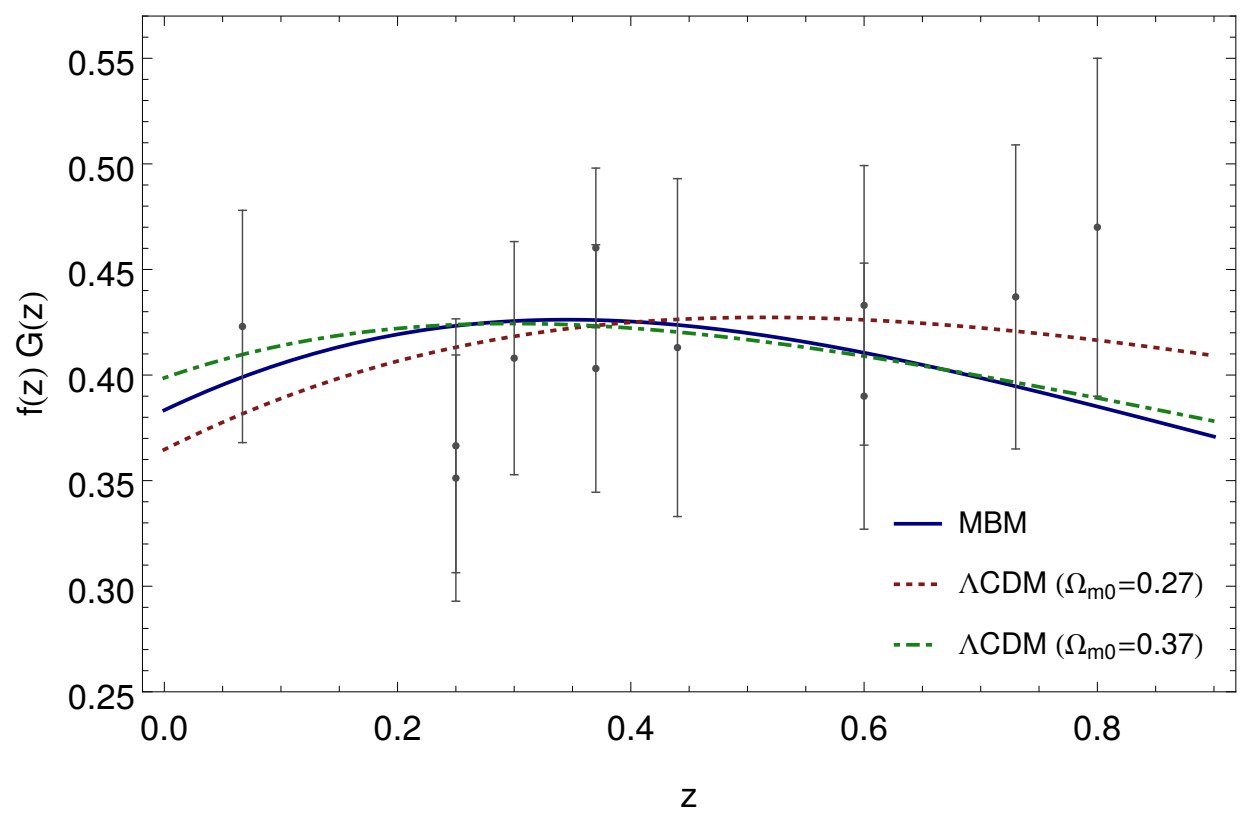

Figure 4: Comparison of growth histories in the MBM with $\beta_{1}=1.39$ (blue) and $\Lambda$ CDM (dotted red: $\Omega_{m 0}=0.27 ;$ dot-dashed green: $\Omega_{m 0}=0.37$ which corresponds to the present matter density in the best-fit MBM). The data points are taken from Ref. [20]. Note that the normalization of the curves is immaterial due to the marginalization over $\sigma_{8}$.

\section{Acknowledgments}

We acknowledge support from DFG through the project TRR33 "The Dark Universe". We thank Mariele Motta for discussions and cross-checks on the perturbation equations and Valerio Marra for help with the supernovae catalog, and Y. Akrami, T. Koivisto, and A. Solomon for discussions on the perturbation equations.

\section{Appendix A: Explicit expressions for the matrices $M_{i j}$ and $N_{i j}$}

In this appendix we will present the elements of the matrices $M_{i j}$ and $N_{i j}$ appearing in the second-order differential equation (34). We start by defining the functions

$$
\begin{aligned}
& K_{1} \equiv K_{2} \equiv \frac{4 r-6 r^{3}}{a^{2}\left(3 \beta_{1} r^{2}+\beta_{1}\right)}, \\
& K_{5} \equiv K_{6} \equiv-\frac{24 k^{2}\left(2-3 r^{2}\right)^{2}}{a^{2} r\left(3 r^{2}+1\right)^{2} \beta_{1}}, \\
& K_{8} \equiv-\frac{12 \sqrt{3} a\left(2-3 r^{2}\right)^{2}\left(3 r^{2}-1\right) \sqrt{\beta_{1}}}{r^{5 / 2}\left(3 k r^{2}+k\right)^{2}}, \\
& K_{10} \equiv-\frac{a^{2} k\left(3 r^{2}-1\right) \beta_{1}}{\sqrt{3}\left(3 r^{3}+r\right)}, \\
& K_{12} \equiv K_{13} \equiv \frac{\sqrt{3} a^{2} k\left(3 r^{2}-1\right) \beta_{1}}{4 r-6 r^{3}}, \\
& K_{16} \equiv \frac{9 r^{2}-6}{k^{2}\left(3 r^{2}+1\right)}, \\
& K_{19} \equiv \frac{a^{2}\left(135 r^{6}+378 r^{4}-171 r^{2}-22\right) \beta_{1}}{8 k^{2}\left(2-3 r^{2}\right)^{2}\left(3 r^{3}+r\right)}, \\
& K_{21} \equiv \frac{a^{2}\left(3 r^{2}+1\right) \beta_{1}}{4 k^{2} r\left(3 r^{2}-2\right)}, \\
& K_{3} \equiv K_{4} \equiv \frac{1}{a^{2} r \beta_{1}}, \\
& K_{7} \equiv-\frac{12\left(2-3 r^{2}\right)^{2}\left(3 r^{2}-1\right)}{\left(3 r^{3}+r\right)^{2}}, \\
& K_{9} \equiv-\frac{a\left(3 r^{2}-1\right) \sqrt{\beta_{1}}}{k \sqrt{r}}, \\
& K_{11} \equiv \frac{4 k\left(3 r^{2}-2\right)}{\sqrt{3}\left(3 r^{2}+1\right)}, \\
& K_{14} \equiv K_{15} \equiv \frac{1}{a^{2} r \beta_{1}}, \\
& K_{17} \equiv K_{18} \equiv \frac{1}{a^{2} r \beta_{1}}+\frac{3}{2 k^{2}}, \\
& K_{20} \equiv-\frac{9 a^{2}\left(1-3 r^{2}\right)^{2}\left(9 r^{2}+2\right) \beta_{1}}{8 k^{2}\left(2-3 r^{2}\right)^{2}\left(3 r^{3}+r\right)}, \\
& K_{22} \equiv-\frac{3 a^{2}\left(3 r^{2}-1\right) \beta_{1}}{4 k^{2} r\left(3 r^{2}-2\right)},
\end{aligned}
$$




$$
\begin{array}{rlrl}
K_{23} & \equiv \frac{1}{\mathcal{H}}, & K_{24} \equiv k^{2}-6 \mathcal{H}^{\prime}, \\
K_{25} \equiv-3 \mathcal{H}^{2}, & K_{26} \equiv-\frac{k^{2}}{\mathcal{H}}, \\
K_{27} \equiv k^{2}\left(\mathcal{H}+\mathcal{H}^{\prime}\right), & K_{28} \equiv k^{2} \mathcal{H}, \\
K_{29} \equiv-\frac{12 k^{2}\left(2-3 r^{2}\right)^{2}}{\left(3 r^{2}+1\right)^{2}}, & K_{30} \equiv-\frac{36\left(2-3 r^{2}\right)^{2}}{\left(3 r^{2}+1\right)^{2}}, \\
K_{31} \equiv \frac{12\left(2-3 r^{2}\right)^{2}\left(3 \beta_{1} a^{2}+2 k^{2} r\right)}{a^{2}\left(3 r^{2}+1\right)^{2} \beta_{1}}, & K_{32} \equiv-\frac{6 a^{2}\left(3 r^{2}-2\right) \beta_{1}}{3 r^{3}+r}, \\
K_{33} \equiv \frac{18 a^{2}\left(3 r^{2}-2\right)\left(3 r^{2}-1\right) \beta_{1}}{r\left(3 r^{2}+1\right)^{2}}, & K_{34} \equiv \frac{4 r-6 r^{3}}{a^{2}\left(3 \beta_{1} r^{2}+\beta_{1}\right)}, \\
K_{35} \equiv \frac{4 r-6 r^{3}}{a^{2}\left(3 \beta_{1} r^{2}+\beta_{1}\right)}, & K_{36} \equiv K_{37} \equiv \frac{1}{a^{2} r \beta_{1}},
\end{array}
$$

which only depend on the background quantities $\beta_{1}, r, \mathcal{H}$ and the wave number $k$. Although we introduced several redundant functions, the definitions of those functions turn out to be useful since in every bimetric model the dependencies of both $M_{i j}$ and $N_{i j}$ on the $K_{i}$ functions are the same. We proceed bywith defining

$$
\begin{aligned}
& L_{1} \equiv K_{13}+\left(3 K_{2} K_{31}^{2}\right)^{-1}\left(3 K_{1} K_{11} K_{31} K_{33}+2 \sqrt{3} k K_{2}\left(K_{33} K_{31}^{\prime}-K_{31} K_{33}^{\prime}\right)\right) \text {, } \\
& L_{2} \equiv\left(3 K_{2} K_{31}^{2}\right)^{-1}\left[2 \sqrt{3} k K_{2}\left(K_{14}\left(K_{31}\left(K_{29}+K_{32}^{\prime}\right)-K_{32} K_{31}^{\prime}\right)-K_{31}\left(K_{30}-2 K_{32} K_{14}^{\prime}\right)\right)\right. \\
& \left.-3 K_{14} K_{31}\left(K_{12} K_{2} K_{31}+K_{1} K_{11} K_{32}\right)\right] \text {, } \\
& L_{3} \equiv\left(3 K_{2} K_{31}^{2}\right)^{-1}\left[2 \sqrt{3} k K_{2}\left(2 K_{31} K_{32} K_{15}^{\prime}+K_{15}\left(K_{31}\left(K_{29}+K_{32}^{\prime}\right)-K_{32} K_{31}^{\prime}\right)\right)\right. \\
& \left.-3 K_{15} K_{31}\left(K_{12} K_{2} K_{31}+K_{1} K_{11} K_{32}\right)\right] \text {, } \\
& L_{4} \equiv-\left(3 K_{2} K_{31}^{2}\right)^{-1}\left[3\left(K_{11} K_{3}+K_{12} K_{2} K_{14}^{\prime}\right) K_{31}^{2}+3 K_{1} K_{11}\left(K_{14} K_{29}-K_{30}+K_{32} K_{14}^{\prime}\right) K_{31}\right. \\
& \left.-2 \sqrt{3} k K_{2}\left(K_{14} K_{29}^{\prime}-K_{30}^{\prime}+K_{14}^{\prime}\left(K_{29}+K_{32}^{\prime}\right)+K_{32} K_{14}^{\prime \prime}\right) K_{31}+2 \sqrt{3} k K_{2}\left(K_{14} K_{29}-K_{30}+K_{32} K_{14}^{\prime}\right) K_{31}^{\prime}\right], \\
& L_{5} \equiv\left(3 K_{2} K_{31}^{2}\right)^{-1}\left[-3 K_{11} K_{4} K_{31}^{2}-3 K_{1} K_{11}\left(K_{15} K_{29}+K_{32} K_{15}^{\prime}\right) K_{31}\right. \\
& \left.+K_{2}\left(2 \sqrt{3} k\left(-K_{32} K_{15}^{\prime} K_{31}^{\prime}+K_{29}\left(K_{31} K_{15}^{\prime}-K_{15} K_{31}^{\prime}\right)+K_{31}\left(K_{15} K_{29}^{\prime}+K_{15}^{\prime} K_{32}^{\prime}+K_{32} K_{15}^{\prime \prime}\right)\right)-3 K_{12} K_{31}^{2} K_{15}^{\prime}\right)\right] \text {, } \\
& L_{6} \equiv-\frac{2 k K_{33}}{\sqrt{3} K_{31}} \\
& L_{7} \equiv \frac{2 k K_{14} K_{32}}{\sqrt{3} K_{31}}, \\
& L_{8} \equiv \frac{2 k K_{15} K_{32}}{\sqrt{3} K_{31}}, \\
& L_{9} \equiv K_{20}+\frac{\left(K_{1} K_{16} k^{2}+3 K_{2}\right) K_{33}}{k^{2} K_{2} K_{31}}, \\
& L_{10} \equiv K_{14}\left(-K_{19}-\frac{\left(K_{1} K_{16} k^{2}+3 K_{2}\right) K_{32}}{k^{2} K_{2} K_{31}}\right)-2 K_{21} K_{14}^{\prime} \text {, } \\
& L_{11} \equiv K_{15}\left(-K_{19}-\frac{\left(K_{1} K_{16} k^{2}+3 K_{2}\right) K_{32}}{k^{2} K_{2} K_{31}}\right)-2 K_{21} K_{15}^{\prime}, \\
& L_{12} \equiv-\left(k^{2} K_{2} K_{31}\right)^{-1}\left[-K_{18} K_{2} K_{31} k^{2}+K_{16} K_{3} K_{31} k^{2}+K_{1} K_{16}\left(K_{14} K_{29}-K_{30}+K_{32} K_{14}^{\prime}\right) k^{2}\right. \\
& \left.+K_{19} K_{2} K_{31} K_{14}^{\prime} k^{2}+K_{2} K_{21} K_{31} K_{14}^{\prime \prime} k^{2}-3 K_{2} K_{30}+K_{14} K_{2}\left(K_{31} k^{2}+3 K_{29}\right)+3 K_{2} K_{32} K_{14}^{\prime}\right] \text {, } \\
& L_{13} \equiv K_{17}-K_{19} K_{15}^{\prime}-K_{21} K_{15}^{\prime \prime}-\left(k^{2} K_{2} K_{31}\right)^{-1}\left[K_{16} K_{31} K_{4} k^{2}\right. \\
& \left.+K_{1} K_{16}\left(K_{15} K_{29}+K_{32} K_{15}^{\prime}\right) k^{2}+K_{15} K_{2}\left(K_{31} k^{2}+3 K_{29}\right)+3 K_{2} K_{32} K_{15}^{\prime}\right] \text {, } \\
& L_{14} \equiv K_{22} \text {, } \\
& L_{15} \equiv-K_{14} K_{21} \text {, } \\
& L_{16} \equiv-K_{15} K_{21} \text {, } \\
& L_{17} \equiv\left[K_{9}\left(K_{7}\left(K_{24} K_{7} K_{9} K_{31}^{2}+\left(K_{31} K_{8} K_{10}^{\prime}+K_{6} K_{9} K_{33}^{\prime}+K_{33} K_{9} K_{6}^{\prime}\right) K_{31}-K_{33} K_{6} K_{9} K_{31}^{\prime}\right)-K_{31} K_{33} K_{6} K_{9} K_{7}^{\prime}\right)\right.
\end{aligned}
$$




$$
\begin{aligned}
& \left.-K_{10} K_{31}^{2}\left(K_{9}\left(K_{23} K_{7}^{2}-K_{8}^{\prime} K_{7}+K_{8} K_{7}^{\prime}\right)+K_{7} K_{8} K_{9}^{\prime}\right)\right]\left(K_{31} K_{7} K_{9}\right)^{-2}, \\
& L_{18} \equiv\left(\sqrt{3} K_{31} K_{7} K_{9}\right)^{-2}\left[-2 \sqrt{3} k K_{31}^{2}\left(K_{9}\left(K_{23} K_{7}^{2}-K_{8}^{\prime} K_{7}+K_{8} K_{7}^{\prime}\right)+K_{7} K_{8} K_{9}^{\prime}\right)+3 K_{9}^{2}\left(K_{14} K_{31} K_{32} K_{6} K_{7}^{\prime}\right.\right. \\
& \left.\left.-K_{7}\left(\left(K_{5}-3 K_{7}\right) K_{31}^{2}+\left(K_{6}\left(-K_{30}+2 K_{32} K_{14}^{\prime}+K_{14}\left(K_{29}+K_{32}^{\prime}\right)\right)+K_{14} K_{32} K_{6}^{\prime}\right) K_{31}-K_{14} K_{32} K_{6} K_{31}^{\prime}\right)\right)\right] \text {, } \\
& L_{19} \equiv\left(3 K_{31}^{2} K_{7}^{2} K_{9}\right)^{-1}\left[-2 \sqrt{3} k K_{7} K_{8} K_{31}^{2}+3 K_{15} K_{32} K_{6} K_{7} K_{9} K_{31}^{\prime}\right. \\
& \left.+3 K_{9}\left(K_{15} K_{32} K_{6} K_{7}^{\prime}-K_{7}\left(2 K_{32} K_{6} K_{15}^{\prime}+K_{15}\left(K_{6}\left(K_{29}+K_{32}^{\prime}\right)+K_{32} K_{6}^{\prime}\right)\right)\right) K_{31}\right], \\
& L_{20} \equiv-\left(K_{31} K_{7}\right)^{-2}\left[K_{7} K_{5}^{\prime} K_{31}^{2}-K_{5} K_{7}^{\prime} K_{31}^{2}+K_{14} K_{6} K_{7} K_{29}^{\prime} K_{31}-K_{6} K_{7} K_{30}^{\prime} K_{31}+K_{6} K_{7} K_{14}^{\prime} K_{32}^{\prime} K_{31}\right. \\
& -K_{30} K_{7} K_{6}^{\prime} K_{31}+K_{32} K_{7} K_{14}^{\prime} K_{6}^{\prime} K_{31}+K_{30} K_{6} K_{7}^{\prime} K_{31}-K_{32} K_{6} K_{14}^{\prime} K_{7}^{\prime} K_{31}+K_{32} K_{6} K_{7} K_{14}^{\prime \prime} K_{31} \\
& \left.+K_{30} K_{6} K_{7} K_{31}^{\prime}-K_{32} K_{6} K_{7} K_{14}^{\prime} K_{31}^{\prime}+K_{29}\left(K_{31}\left(K_{6} K_{7} K_{14}^{\prime}+K_{14} K_{7} K_{6}^{\prime}-K_{14} K_{6} K_{7}^{\prime}\right)-K_{14} K_{6} K_{7} K_{31}^{\prime}\right)\right] \text {, } \\
& L_{21} \equiv\left(\sqrt{3} K_{31} K_{7} K_{9}\right)^{-2}\left[2 \sqrt{3} k K_{31}^{2}\left(K_{9}\left(K_{23} K_{7}^{2}-K_{8}^{\prime} K_{7}+K_{8} K_{7}^{\prime}\right)+K_{7} K_{8} K_{9}^{\prime}\right)\right. \\
& -3 K_{9}^{2}\left(K_{15} K_{31} K_{6} K_{7} K_{29}^{\prime}+K_{29}\left(K_{31}\left(K_{6} K_{7} K_{15}^{\prime}+K_{15} K_{7} K_{6}^{\prime}-K_{15} K_{6} K_{7}^{\prime}\right)-K_{15} K_{6} K_{7} K_{31}^{\prime}\right)\right. \\
& \left.\left.+K_{15}^{\prime}\left(K_{31} K_{6} K_{7} K_{32}^{\prime}+K_{32}\left(K_{31} K_{7} K_{6}^{\prime}-K_{6}\left(K_{7} K_{31}^{\prime}+K_{31} K_{7}^{\prime}\right)\right)\right)+K_{31} K_{32} K_{6} K_{7} K_{15}^{\prime \prime}\right)\right] \text {, } \\
& L_{22} \equiv K_{25}+K_{7}^{-1}\left(\frac{K_{33} K_{6}}{K_{31}}+\frac{K_{10} K_{8}}{K_{9}}\right) \text {, } \\
& L_{23} \equiv\left(3 K_{7}\right)^{-1}\left(\frac{2 \sqrt{3} k K_{8}}{K_{9}}-\frac{3 K_{14} K_{32} K_{6}}{K_{31}}\right) \text {, } \\
& L_{24} \equiv-\frac{K_{15} K_{32} K_{6}}{K_{31} K_{7}}, \\
& L_{25} \equiv K_{26}+\frac{2 k\left(K_{9}-K_{9}^{\prime}\right)}{\sqrt{3} K_{9}^{2}}, \\
& L_{26} \equiv \frac{K_{10} K_{9}^{\prime}-K_{9}\left(K_{10}-K_{27} K_{9}+K_{10}^{\prime}\right)}{K_{9}^{2}}, \\
& L_{27} \equiv \frac{2 k\left(K_{9}^{\prime}-K_{9}\right)}{\sqrt{3} K_{9}^{2}}, \\
& L_{28} \equiv \frac{2 k}{\sqrt{3} K_{9}} \text {, } \\
& L_{29} \equiv K_{28}-\frac{K_{10}}{K_{9}}, \\
& L_{30} \equiv-\frac{2 k}{\sqrt{3} K_{9}},
\end{aligned}
$$

and

$$
\begin{aligned}
& G_{1} \equiv \frac{-L_{12} L_{23} L_{8}+L_{12} L_{24} L_{7}+L_{15} L_{20} L_{8}-L_{15} L_{24} L_{4}-L_{16} L_{20} L_{7}+L_{16} L_{23} L_{4}}{-L_{1} L_{15} L_{24}+L_{1} L_{16} L_{23}+L_{15} L_{17} L_{8}-L_{16} L_{17} L_{7}-L_{23} L_{8} L_{9}+L_{24} L_{7} L_{9}}, \\
& G_{2} \equiv \frac{-L_{13} L_{23} L_{8}+L_{13} L_{24} L_{7}+L_{15} L_{21} L_{8}-L_{15} L_{24} L_{5}-L_{16} L_{21} L_{7}+L_{16} L_{23} L_{5}}{-L_{1} L_{15} L_{24}+L_{1} L_{16} L_{23}+L_{15} L_{17} L_{8}-L_{16} L_{17} L_{7}-L_{23} L_{8} L_{9}+L_{24} L_{7} L_{9}}, \\
& G_{3} \equiv \frac{-L_{10} L_{23} L_{8}+L_{10} L_{24} L_{7}+L_{15} L_{18} L_{8}-L_{15} L_{2} L_{24}-L_{16} L_{18} L_{7}+L_{16} L_{2} L_{23}}{-L_{1} L_{15} L_{24}+L_{1} L_{16} L_{23}+L_{15} L_{17} L_{8}-L_{16} L_{17} L_{7}-L_{23} L_{8} L_{9}+L_{24} L_{7} L_{9}}, \\
& G_{4} \equiv \frac{-L_{11} L_{23} L_{8}+L_{11} L_{24} L_{7}+L_{15} L_{19} L_{8}-L_{15} L_{24} L_{3}-L_{16} L_{19} L_{7}+L_{16} L_{23} L_{3}}{-L_{1} L_{15} L_{24}+L_{1} L_{16} L_{23}+L_{15} L_{17} L_{8}-L_{16} L_{17} L_{7}-L_{23} L_{8} L_{9}+L_{24} L_{7} L_{9}} .
\end{aligned}
$$

The elements of the matrices $M_{i j}$ and $N_{i j}$ are then given by

$$
\begin{aligned}
M_{11}= & \frac{L_{11}-G_{4} L_{9}-L_{14}\left(G_{2}+G_{4}^{\prime}\right)+\left(L_{23}-G_{3} L_{22}\right)^{-1}\left(G_{3} L_{14}-L_{15}\right)\left(-G_{4} L_{17}+L_{19}-L_{22}\left(G_{2}+G_{4}^{\prime}\right)\right)}{-G_{4} L_{14}+L_{16}+\left(L_{23}-G_{3} L_{22}\right)^{-1}\left(G_{3} L_{14}-L_{15}\right)\left(L_{24}-G_{4} L_{22}\right)} \\
M_{12}= & \frac{L_{10}-G_{3} L_{9}-L_{14}\left(G_{1}+G_{3}^{\prime}\right)+\left(L_{23}-G_{3} L_{22}\right)^{-1}\left(G_{3} L_{14}-L_{15}\right)\left(-G_{3} L_{17}+L_{18}-L_{22}\left(G_{1}+G_{3}^{\prime}\right)\right)}{-G_{4} L_{14}+L_{16}+\left(L_{23}-G_{3} L_{22}\right)^{-1}\left(G_{3} L_{14}-L_{15}\right)\left(L_{24}-G_{4} L_{22}\right)}, \\
M_{21}= & \left(L_{16}\left(L_{23}-G_{3} L_{22}\right)+L_{15}\left(G_{4} L_{22}-L_{24}\right)+L_{14}\left(G_{3} L_{24}-G_{4} L_{23}\right)\right)^{-1}\left[L_{14} L_{17} G_{4}^{2}-L_{22} L_{9} G_{4}^{2}-L_{14} L_{19} G_{4}\right. \\
& \left.+L_{24} L_{9} G_{4}+L_{11}\left(G_{4} L_{22}-L_{24}\right)+G_{2} L_{14} L_{24}+L_{14} L_{24} G_{4}^{\prime}+L_{16}\left(-G_{4} L_{17}+L_{19}-L_{22}\left(G_{2}+G_{4}^{\prime}\right)\right)\right],
\end{aligned}
$$




$$
\begin{aligned}
M_{22}= & \left(L_{16}\left(G_{3} L_{22}-L_{23}\right)+L_{15}\left(L_{24}-G_{4} L_{22}\right)+L_{14}\left(G_{4} L_{23}-G_{3} L_{24}\right)\right)^{-1}\left[-G_{3} G_{4} L_{14} L_{17}+G_{4} L_{14} L_{18}\right. \\
& \left.-G_{1} L_{14} L_{24}+L_{10}\left(L_{24}-G_{4} L_{22}\right)+G_{3} G_{4} L_{22} L_{9}-G_{3} L_{24} L_{9}-L_{14} L_{24} G_{3}^{\prime}+L_{16}\left(G_{3} L_{17}-L_{18}+L_{22}\left(G_{1}+G_{3}^{\prime}\right)\right)\right]
\end{aligned}
$$

and

$$
\begin{aligned}
N_{11}= & \frac{L_{13}-G_{2} L_{9}-L_{14} G_{2}^{\prime}+\left(L_{23}-G_{3} L_{22}\right)^{-1}\left(G_{3} L_{14}-L_{15}\right)\left(-G_{2} L_{17}+L_{21}-L_{22} G_{2}^{\prime}\right)}{-G_{4} L_{14}+L_{16}+\left(L_{23}-G_{3} L_{22}\right)^{-1}\left(G_{3} L_{14}-L_{15}\right)\left(L_{24}-G_{4} L_{22}\right)}, \\
N_{12}= & \frac{L_{12}-G_{1} L_{9}-L_{14} G_{1}^{\prime}+\left(L_{23}-G_{3} L_{22}\right)^{-1}\left(G_{3} L_{14}-L_{15}\right)\left(-G_{1} L_{17}+L_{20}-L_{22} G_{1}^{\prime}\right)}{-G_{4} L_{14}+L_{16}+\left(L_{23}-G_{3} L_{22}\right)^{-1}\left(G_{3} L_{14}-L_{15}\right)\left(L_{24}-G_{4} L_{22}\right)}, \\
N_{21}= & \left(L_{16}\left(L_{23}-G_{3} L_{22}\right)+L_{15}\left(G_{4} L_{22}-L_{24}\right)+L_{14}\left(G_{3} L_{24}-G_{4} L_{23}\right)\right)^{-1}\left[G_{2} G_{4} L_{14} L_{17}-G_{4} L_{14} L_{21}\right. \\
& \left.+L_{13}\left(G_{4} L_{22}-L_{24}\right)-G_{2} G_{4} L_{22} L_{9}+G_{2} L_{24} L_{9}+L_{14} L_{24} G_{2}^{\prime}+L_{16}\left(-G_{2} L_{17}+L_{21}-L_{22} G_{2}^{\prime}\right)\right], \\
N_{22}= & \left(L_{16}\left(L_{23}-G_{3} L_{22}\right)+L_{15}\left(G_{4} L_{22}-L_{24}\right)+L_{14}\left(G_{3} L_{24}-G_{4} L_{23}\right)\right)^{-1}\left[G_{1} G_{4} L_{14} L_{17}-G_{4} L_{14} L_{20}\right. \\
& \left.+L_{12}\left(G_{4} L_{22}-L_{24}\right)-G_{1} G_{4} L_{22} L_{9}+G_{1} L_{24} L_{9}+L_{14} L_{24} G_{1}^{\prime}+L_{16}\left(-G_{1} L_{17}+L_{20}-L_{22} G_{1}^{\prime}\right)\right] .
\end{aligned}
$$

[1] K. Hinterbichler. Reviews of Modern Physics 84, 671 (2012). 1105.3735.

[2] C. de Rham. ArXiv e-prints (2014). 1401.4173.

[3] M. Maggiore and M. Mancarella (2014). 1402.0448.

[4] C. de Rham and G. Gabadadze. Physics Letters B 693, 334 (2010). 1006.4367.

[5] C. de Rham and G. Gabadadze. Phys. Rev. D 82, 044020 (2010). 1007.0443.

[6] C. de Rham and L. Heisenberg. Phys. Rev. D 84, 043503 (2011). 1106.3312.

[7] C. de Rham, G. Gabadadze, and A. J. Tolley. Physical Review Letters 106, 231101 (2011). 1011.1232.

[8] S. F. Hassan and R. A. Rosen. Journal of High Energy Physics 2, 126 (2012). 1109.3515.

[9] S. F. Hassan, R. A. Rosen, and A. Schmidt-May. Journal of High Energy Physics 2, 26 (2012). 1109.3230.

[10] Y. Akrami, T. S. Koivisto, D. F. Mota, and M. Sandstad. JCAP 10, 046 (2013). 1306.0004.

[11] M. von Strauss, et al. Journal of Cosmology and Astroparticle Physics 2012, 042 (2012). URL http://stacks.iop.org/ $1475-7516 / 2012 / i=03 / a=042$

[12] Y. Akrami, T. S. Koivisto, and M. Sandstad. Journal of High Energy Physics 3, 99 (2013). 1209.0457.

[13] D. Comelli, M. Crisostomi, and L. Pilo. JHEP 1206, 085 (2012). 1202.1986.

[14] A. De Felice, T. Nakamura, and T. Tanaka. ArXiv e-prints (2013). 1304.3920.

[15] D. Comelli, M. Crisostomi, F. Nesti, and L. Pilo. Journal of High Energy Physics 3, 67 (2012). 1111.1983.

[16] M. S. Volkov. Journal of High Energy Physics 1, 35 (2012). 1110.6153.

[17] M. Berg, et al. JCAP 12, 021 (2012). 1206.3496.

[18] F. Koennig, A. Patil, and L. Amendola. Journal of Cosmology and Astroparticle Physics 2014, 029 (2014). 1312.3208, URL http://stacks .iop.org/1475-7516/2014/i=03/a=029.

[19] A. De Felice, et al. (2014). 1404.0008.

[20] E. Macaulay, I. K. Wehus, and H. K. Eriksen (2013). 1303.6583.

[21] M. Fasiello and A. J. Tolley. JCAP 12, 002 (2013). 1308.1647.

[22] H. van Dam and M. Veltman. Nuclear Physics B 22, 397 (1970). URL http://dx.doi.org/10.1016/0550-3213(70) 90416-5.

[23] V. I. Zakharov. Soviet Journal of Experimental and Theoretical Physics Letters 12, 312 (1970).

[24] G. W. Horndeski. Int.J.Th.Phys. 10, 363 (1974).

[25] A. De Felice, T. Kobayashi, and S. Tsujikawa. Phys.Lett. B706, 123 (2011). 1108.4242.

[26] L. Amendola, et al. Phys. Rev. D 87, 023501 (2013). 1210.0439.

[27] O. Lahav, P. B. Lilje, J. R. Primack, and M. J. Rees. Mon.Not.Roy.Astron.Soc. 251, 128 (1991).

[28] R. Laureijs, et al. ArXiv e-prints (2011). 1110.3193.

[29] A. Piloyan, V. Marra, M. Baldi, and L. Amendola (2014). 1401.2656.

[30] F. Beutler, et al. (2012). 1204.4725.

[31] L. Samushia, W. J. Percival, and A. Raccanelli. Mon.Not.Roy.Astron.Soc. 420, 2102 (2012). 1102.1014.

[32] R. Tojeiro, et al. (2012). 1203.6565.

[33] C. Blake, et al. Mon.Not.Roy.Astron.Soc. 425, 405 (2012). 1204.3674.

[34] S. de la Torre, et al. (2013). 1303.2622.

[35] E. Komatsu, et al. The Astrophysical Journal 192, 18 (2011). 1001.4538.

[36] W. J. Percival, et al. Mon.Not.Roy.Astron.Soc. 401, 2148 (2010). 0907.1660. 